\title{
Terminal shape and velocity of a rising bubble by phase-field-based
}

\section{incompressible Lattice Boltzmann model}

\author{
Feng Ren ${ }^{1,2}$, Baowei Song ${ }^{1}$, Michael C. Sukop ${ }^{2, *}$ \\ 1 School of Marine Science and Technology, Northwestern Polytechnical University, Xi'an, Shaanxi 710072, China \\ 2 Department of Earth and Environment, Florida International University, 11200 SW 8th Street, Miami, FL 33199, USA
}

\begin{abstract}
This article describes the simulation of three-dimensional buoyancy-driven bubble rise using a phase-field-based incompressible Lattice Boltzmann model. The effect of the Cahn-Hilliard mobility parameter, which is the rate of diffusion relaxation from non-equilibrium toward equilibrium state of chemical potential, is evaluated in detail. In contrast with previous work that pursues a high density ratio of binary fluids in the hydrodynamic equation, we apply a large dynamic viscosity ratio, together with a matched density pair and a separate compensating gas phase buoyant force, and the numerical results fit previous experimental results well. Through analysis, it is noted that for cases with moderate Reynolds number, a large value of mobility keeps a relatively sharp interface, while smaller values of mobility would result in diffusive interfacial regions. Moreover, for cases with large Reynolds number, small bubbles at the tail tend to separate more easily when the value of mobility is larger. This article offers some potentially useful details for performing phase-field-based simulations.
\end{abstract}

Key words: bubble rise; Lattice Boltzmann method; phase-field model; Cahn-Hilliard mobility

\section{Introduction}

Multiphase flows are common phenomena and are of great significance in both scientific and engineering fields; bubble rising processes are typical flows of interest. Water resources-related problems include for example, methane and carbon dioxide ebullition in thawing high-latitude peat soils and bubble-based hazardous waste cleanup efforts at the U.S. Department of Energy's Hanford Site. There is also potential for liberation and release of gas bubbles into groundwater as a result of hydraulic fracturing. In different flow regimes, the shape and velocity of the rising bubble will be quite different [1,2], typically including oblate ellipsoidal, oblate ellipsoidal disk, oblate ellipsoidal cap, spherical cap, skirted, etc. A deeper understanding of the dynamic characteristic of a rising bubble can enable promising applications, e.g., bubble rise in porous media [3]. As a complement to experimental investigations that measure instantaneous bubble velocity and record changing bubble shape [1,2,4], numerical simulation methods may offer an alternative choice for developing such understanding [5-7].

For a numerical multiphase model, the key factor is to precisely track the interface and model the interfacial interaction, which can be achieved by solving a phase-tracking equation and modeling the interfacial tension force in the hydrodynamic equation. Typical methods to these problems include the volume of fluid (VOF) method [8], the level-set method (LSM) [9], phase-field-based models [10,11], etc.

Among these models, phase field theory is often adopted by researchers from scientific and engineering fields, and the most typical phase-tracking equation is the Cahn-Hilliard $(\mathrm{CH})$ equation [12]. The $\mathrm{CH}$ equation is an equation of mathematical physics, describing spontaneously separated binary phases. Different from the temporal propagation and convection treatment in VOF and LSM, a diffusion term is also incorporated, where chemical potential and mobility are applied. Herein mobility is introduced as the rate of diffusion relaxation from non-equilibrium toward equilibrium state of chemical potential [13]. Although the phase field models have been solved and applied by many scholars, the physical meaning of mobility has not been fully understood, beyond its effect on the simulation results $[13,14]$.

To solve the $\mathrm{CH}$ equation, conventional methods, including finite difference methods (FDM), finite

*Corresponding author. Email: sukopm@fiu.edu.

(C) 2016. This manuscript version is made available under the Elsevier user license

http://www.elsevier.com/open-access/userlicense/1.0/ 
volume methods (FVM), and finite element methods (FEM) [15], can be applied, each with advantages and drawbacks. In recent years, the Lattice Boltzmann method (LBM) has been developed to solve the $\mathrm{CH}$ equation and also the hydrodynamic equation [6,16-20]. It has drawn much attention due to its kinetic-based background, simple algorithm, and great performance in parallel computing. Single-relaxation time (SRT) and multi-relaxation time (MRT) schemes are two main branches of LBM, and the formulation of the MRT scheme is more general and stable. The difference lies in the choice of the relaxation times (described below), and if the elements of the matrix of the relaxation times in the MRT scheme are set to be the same, then it is reduced to the SRT scheme. In addition, other multiphase models based on LBM have been used for some time and include: the Rothman-Keller (color-gradient) model [21], the Shan-Chen (pseudopotential) model [22,23], the free-energy-based model [24], the He-Chen-Zhang model [25,26], etc.

Numerous investigations have been done to enhance understanding of the bubble rising process in the past. Clift et al.[1] and Bhaga et al.[2], respectively, conducted hydrodynamic experiments and measured the terminal shape, terminal velocity, and the wake behind the bubble. Hua et al. simulated the single-bubble and multi-bubble rising process using VOF [27] and LBM [28,29]. Huang et al. developed the axisymmetric model [30] based on the model of He et al.[25,26], and applied their model to simulate bubble rising processes. Ngachin et al. simulated the bubble rising process using a multicomponent Shan-Chen model based on their effective buoyancy method [31]. Amaya-Bower et al. used the CH equation and simulated bubble rising process under moderate Reynolds number [32]. In addition, similar numerical work is conducted for simulating falling droplet processes [33,34]. However, the above simulations share the same drawback of not being able to recover the correct incompressible continuity equation and operating in a compressible regime [30,35], which can make the results deviate from real situations in cases where the Reynolds number is high or cases where the deformation of bubbles/droplets is large. In problems involving bubble dynamics, another widely used approach is the boundary element method (BEM) [36-38], where the mesh is constructed over the modelled surface, thus greatly improving the computational efficiency. However, in the calculation, the viscosity and interfacial tension is usually ignored, which works in situations where the Reynolds number is extremely high.

The latest phase field model under the framework of LBM has been capable of correctly recovering the $\mathrm{CH}$ equation through the Chapman-Enskog analysis [17-19]. Moreover, the LB hydrodynamic equation has overcome the drawback of near-incompressible limit [17-19]. But in practice, the effect of mobility is often ignored, sometimes making the simulations deviate from real situations.

The aim of this work is to reveal the ability of the incompressible model to deal with the common bubble rising problem, and to present a direct investigation into the effect of the Cahn-Hilliard mobility. In our work, a flow regime with moderate Reynolds number is simulated to enable comparisons with previous experimental and numerical investigations. Gas bubbles can be considered relatively incompressible under certain conditions (for example, an ideal gas bubble rising through one meter of water will change its radius by only 3\%); under other circumstances, an incompressible model like the one we use here would be most appropriate for lower-compressibility rising droplets, such as oil in water.

We outline this article as follows: in Sec. 2, we introduce the framework of the numerical model; in Sec. 3, we validate the model by conducting layered Poiseuille flow simulations; in Sec. 4, we perform bubble rising simulations and present our analysis; in Sec. 5, conclusions are drawn. The simulations are performed using an Intel Xeon CPU and an Nvidia K20m GPU, wherein the former acts as the control node and the latter executes almost all the calculation work.

\section{Methodology}

\subsection{Phase tracking equation}

By applying the phase-field theory, the interface can be captured using the order parameter, which is $\phi_{l}$ for the liquid phase, and $\phi_{g}$ for the gas phase, respectively. And the bulk free energy of an isothermal 
liquid-gas system takes the double-well form [6]

$E_{f} \approx \beta\left(\phi-\phi_{l}\right)^{2}\left(\phi-\phi_{g}\right)^{2}$,

where $\beta$ is a parameter dependent on interfacial thickness and surface tension. The chemical potential can then be derived as

$\mu(\phi)=\mu_{0}(\phi)-\kappa \nabla^{2} \phi=4 \beta\left(\phi-\phi_{l}\right)\left(\phi-\phi_{g}\right)\left(\phi-\frac{\phi_{l}+\phi_{g}}{2}\right)-\kappa \nabla^{2} \phi$,

where $\beta$ and $\kappa$ are related to surface tension, and the relationships are expressed as

$\beta=\frac{12 \sigma}{W\left(\phi_{l}-\phi_{g}\right)^{4}}$,

$\kappa=\frac{3 \sigma W}{2\left(\phi_{l}-\phi_{g}\right)^{2}}$,

where $W$ is the interface width, and $\sigma$ is surface tension.

By solving $\mu(\phi)=0$, the analytical one-dimensional interfacial profile of the order parameter (along the $\mathrm{z}$ direction perpendicular to the interface) can be obtained:

$\phi(z)=\frac{\phi_{l+} \phi_{g}}{2}+\frac{\phi_{l-} \phi_{g}}{2} \tanh \left(\frac{2 z}{W}\right)$.

In our simulations, Eq.5 is also used to initialize the values of order parameter along the normal direction of the interface.

Typically the diffusion-driven Cahn-Hilliard equation is used to track the interfaces of binary flow systems, and is formulated as

$\partial_{t} \phi+\nabla \cdot(\phi \mathbf{u})=\nabla\left(M_{\phi} \nabla \mu\right)$

where $M_{\phi}$ is the mobility. To obtain a non-dimensional form of Eq. 6, we define the dimensionless variables as

$\bar{x}=\frac{x}{x^{*}}, \bar{t}=\frac{t}{t^{*}}, \bar{u}=\frac{u t^{*}}{x^{*}}, \bar{\phi}=\frac{\phi}{\phi_{l}-\phi_{g}}$.

where $x^{*}$ and $t^{*}$ are the reference length and the reference time, respectively. Hence, Eq. 6 can be rewritten as

$\partial_{\bar{t}} \bar{\phi}+\bar{\nabla} \cdot(\phi \mathbf{u})=\bar{\nabla}\left\{\frac{1}{P e} \bar{\nabla}\left[\frac{48}{C h}\left(\bar{\phi}-\bar{\phi}_{l}\right)\left(\bar{\phi}-\bar{\phi}_{l}\right)\left(\bar{\phi}-\frac{\bar{\phi}_{l}+\bar{\phi}_{g}}{2}\right)-\frac{3 C h}{2} \bar{\nabla}^{2} \bar{\phi}\right]\right\}$.

where $P e=\frac{x^{* 3}\left(\phi_{l}-\phi_{g}\right)^{2}}{M_{\phi} \sigma t^{*}}$ is the Péclet number, and $C h=\frac{W}{x^{*}}$ is the Cahn number. These two parameters dominate the phase-tracking process.

Following the description of Liang et al. $[17,18]$, the LB equation with MRT scheme for the $\mathrm{CH}$ equation has the following form

$\mathbf{f}\left(\mathbf{x}+\mathbf{e}_{\mathrm{i}} \delta \mathrm{t}, \mathrm{t}+\delta \mathrm{t}\right)-\mathbf{f}(\mathbf{x}, \mathrm{t})=-\left(\mathbf{M}^{-1} \Lambda^{\mathrm{f}} \mathbf{M}\right)\left[\mathbf{f}(\mathbf{x}, \mathrm{t})-\mathbf{f}^{\mathrm{eq}}(\mathbf{x}, \mathrm{t})\right]+\left[\mathbf{M}^{-1}\left(\mathbf{I}-\frac{\Lambda^{\mathrm{f}}}{2}\right) \mathbf{M}\right] \mathbf{R}$.

where $\mathbf{M}$ is the transformation matrix from velocity space to moment space, and as described in [39]. With the three-dimension and fifteen-speed (D3Q15) scheme, the lattice vectors are

$\mathbf{e}=c\left[\begin{array}{ccccccccccccccc}0 & 1 & -1 & 0 & 0 & 0 & 0 & 1 & -1 & 1 & -1 & 1 & -1 & 1 & -1 \\ 0 & 0 & 0 & 1 & -1 & 0 & 0 & 1 & 1 & -1 & -1 & 1 & 1 & -1 & -1 \\ 0 & 0 & 0 & 0 & 0 & 1 & -1 & 1 & 1 & 1 & 1 & -1 & -1 & -1 & -1\end{array}\right]$,

where $c=\delta x / \delta t$ is lattice speed, and $\delta x=1 l u$ and $\delta t=1 t s$ are lattice unit length and lattice time step, respectively. Herein $l u$ and $t s$ represent the lattice unit of length and time step [5], respectively, and in addition, $m u$ is the lattice mass unit. The equilibrium function is 
$f_{i}^{e q}=\left\{\begin{array}{l}\eta \mu\left(w_{i}-1\right)+\phi, i=0 \\ w_{i} \eta \mu+w_{i} \frac{\boldsymbol{e}_{i} \cdot \phi \boldsymbol{u}}{c_{s}^{2}}, i \neq 0\end{array}\right.$,

where $w_{i}$ are the weighing factors, and they are $w_{0}=2 / 9, w_{1-6}=1 / 9$, and $w_{7-14}=1 / 72$.

The parameter $c_{s}=1 / \sqrt{3} \mathrm{lu} / \mathrm{ts}$ is lattice sound speed. To recover the correct form of the $\mathrm{CH}$ equation, $\mathbf{R}$ in the source term is formulated as

$R_{i}=\frac{w_{i} \mathbf{e}_{i} \cdot \partial_{t}(\phi \mathbf{u})}{c_{s}^{2}}$.

The order parameter is calculated as

$\phi=\sum_{i} f_{i}$.

The MRT models used for both interface tracking $(\mathrm{CH})$ and hydrodynamic equations employ a relaxation time matrix. The relaxation times $s_{3}^{f}=s_{5}^{f}=s_{7}^{f}=1 / \tau^{f}$ are required to ensure the derivation of the correct $\mathrm{CH}$ equation via the Chapman-Enskog expansion, and the remaining relaxation times mainly affect the numerical stability [40,41]. If every relaxation time is set to be $1 / \tau^{\mathrm{f}}$, then the scheme is reduced to the SRT scheme. The situation in dealing with the hydrodynamic equation is quite similar, where the relaxation times have to be set as $s_{9}^{g}=s_{10}^{g}=s_{11}^{g}=s_{12}^{g}=s_{13}^{g}=1 / \tau^{g}$ in order to recover the correct hydrodynamic equation. In fact, so far very few criteria concerning the choice of the free relaxation times have been introduced in the LBM community, especially for the D3Q15 scheme. Here our choices of relaxation times are based on the following considerations: first, keep relaxation times needed to recover the correct governing equations; second, in a simple way, choose uniform values of the remaining relaxation times to enhance numerical stability, and herein they are set to be 1.0 for simplicity.

By Chapman-Enskog expansion with an accuracy of $O\left(\delta t^{2}\right)$, mobility has the following form $M_{\phi}=\eta c_{S}^{2}\left(\tau^{f}-0.5\right) \delta t$,

where $\eta$ is an independent constant introduced to permit the tuning of the mobility.

\subsection{Hydrodynamic equation}

The incompressible Navier-Stokes equations, including the continuity equation, are expressed as

$$
\left\{\begin{array}{c}
\nabla \cdot \mathbf{u}=0 \\
\rho\left(\frac{\partial \mathbf{u}}{\partial t}+\mathbf{u} \cdot \nabla \mathbf{u}\right)=-\nabla p+\nabla \cdot\left[\rho v\left(\nabla \mathbf{u}+\nabla \mathbf{u}^{T}\right)\right]+\mathbf{F}_{S}+\mathbf{F}_{B}
\end{array}\right.
$$

where $\mathbf{F}_{S}$ is the interfacial tension force, and $\mathbf{F}_{B}$ is the buoyant force. Different from some previous models that recover deviated NS equations, which commonly includes a compressible continuity equation and an artificial interfacial force in the hydrodynamic equation [25,26], we apply the latest model by Liang et $a l .[17,18]$, which is proven to be capable of correctly recovering the above NS equations to an accuracy of $O\left(\delta t^{2}+\delta t M a^{2}\right)$, where $M a$ denotes the Mach number. A similar model that recovers the incompressible NS equations can be found in the work of $\mathrm{Zu}$ et al.[19], which, by contrast, contains implicit calculation of pressure. Herein the LB equation with MRT scheme has the following for

$$
\mathbf{g}\left(\mathbf{x}+\mathbf{e}_{\mathrm{i}} \delta \mathrm{t}, \mathrm{t}+\delta \mathrm{t}\right)-\mathbf{g}(\mathbf{x}, \mathrm{t})=-\left(\mathbf{M}^{-1} \Lambda^{\mathrm{g}} \mathbf{M}\right)\left[\mathbf{g}(\mathbf{x}, \mathrm{t})-\mathbf{g}^{\mathrm{eq}}(\mathbf{x}, \mathrm{t})\right]+\left[\mathbf{M}^{-1}\left(\mathbf{I}-\frac{\Lambda^{\mathrm{g}}}{2}\right) \mathbf{M}\right] \mathbf{S} .
$$

The equilibrium functions are

$$
g_{i}^{e q}=\left\{\begin{array}{ll}
\frac{p}{c_{s}^{2}}\left(w_{i}-1\right)+\rho s_{i}(\mathbf{u}), i=0 \\
\frac{p}{c_{s}^{2}} w_{i}+\rho s_{i}(\mathbf{u}), & i \neq 0
\end{array},\right.
$$

where $s_{i}(\mathbf{u})$ is calculated as

$$
s_{i}(\mathbf{u})=w_{i}\left(\frac{\mathbf{e}_{i} \mathbf{u}}{c_{S}^{2}}+\frac{\left(\mathbf{e}_{i} \mathbf{u}\right)^{2}}{2 c_{s}^{4}}-\frac{\mathbf{u}^{2}}{2 c_{S}^{2}}\right) .
$$


The source term $\mathbf{S}$ is formulated as

$S_{i}=\frac{\mathbf{e}_{i}-\mathbf{u}}{c_{s}^{2}}\left\{s_{i}(\mathbf{u}) \cdot \nabla\left(\rho c_{s}^{2}\right)+\left[s_{i}(\mathbf{u})+w_{i}\right] \cdot\left(\mathbf{F}_{s}+\mathbf{F}_{B}+\mathbf{F}_{a}\right)\right\}$

where $\mathbf{F}_{a}$ is introduced to eliminate the artificial interfacial force. This compensating force is proposed by $\mathrm{Li}$ et al. [42] and is expressed as

$\mathbf{F}_{a}=d_{\phi} \rho \cdot\left(M_{\phi} \nabla \mu\right) \mathbf{u}$.

To calculate the interfacial tension force, three forms are usually used. The first scheme [17] uses the chemical potential and the spatial derivative of the order parameter, by setting

$\mathbf{F}_{S}^{(1)}=\mu \nabla \phi=-\kappa \nabla^{2} \phi \nabla \phi+\mu_{0} \nabla \phi$.

The second scheme [19] uses the derivative of chemical potential and the order parameter, i.e.

$\mathbf{F}_{s}^{(2)}=-\phi \nabla \mu=\kappa \phi \nabla \nabla^{2} \phi-\phi \nabla \mu_{0}$.

Note that the above schemes are developed from the original phase-field theory that usually ignores $\mu_{0}$ or its first-order derivatives in the calculation of interfacial tension force [11]. In the work of Chao et al. [35] that applies the HCZ model, it is noted that a wiggle will appear over the interfacial region, which would cause an error in the direction of interfacial force. Thus another interfacial tension calculation scheme is recommended by Kim et al. [11,43] as

$\mathbf{F}_{S}^{(3)}=-\kappa \nabla \cdot\left(\frac{\nabla \phi}{|\nabla \phi|}\right)|\nabla \phi| \nabla \phi$.

Since the third scheme contains the calculation of divergence, on the basis of Eq. 23, we have

$\nabla^{2} \phi=\nabla \cdot(\nabla \phi)=\nabla \cdot\left(\frac{\nabla \phi}{|\nabla \phi|}|\nabla \phi|\right)=|\nabla \phi| \nabla \cdot\left(\frac{\nabla \phi}{|\nabla \phi|}\right)+\frac{\nabla \phi}{|\nabla \phi|} \nabla \cdot(|\nabla \phi|)$, and

$\nabla \phi \frac{\nabla \phi}{|\nabla \phi|} \nabla \cdot(|\nabla \phi|)=|\nabla \phi| \nabla \cdot(|\nabla \phi|)=\frac{1}{2} \nabla \cdot\left(|\nabla \phi|^{2}\right)$.

Hence, a new form can be derived as

$\mathbf{F}_{S}=-\kappa \nabla^{2} \phi \nabla \phi+\frac{\kappa}{2} \nabla \cdot\left(|\nabla \phi|^{2}\right)$.

Eq. 25 is used in the remainder of the article to calculate interfacial tension force. The buoyant force is added only to the gas phase, as in the work of Ngachin et al. [31], and for each node, it is calculated as

$\mathbf{F}_{B}=-\rho_{l} \boldsymbol{g} \mathrm{V}_{i}$,

where $\boldsymbol{g}$ is gravitational acceleration, and $\mathrm{V}_{i}$ is the lattice volume. The buoyant force is actually $\mathbf{F}_{B}=$ $-\left(\rho_{l}-\rho_{g}\right) \mathbf{g V}_{i}$, but it is quite reasonable to approximate it as $\mathbf{F}_{B}=-\rho_{l} \mathbf{g V}_{i}$ because $\rho_{g}$ is often so small in comparison to $\rho_{l}$. In real bubble rising situations, the density ratio of the system is usually very high (water/air pair is around 800), and buoyancy can be approximated using Eq. 26 with little loss of accuracy. To compare with previous experimental work, Eq. 26, together with a high dynamic viscosity ratio, appears to be sufficient for simulating real situations, even though a matched density ratio is used in the hydrodynamic equation and the buoyant force is added separately as described below.

The macroscopic variables have the following explicit form

$\mathbf{u}=\frac{\sum \boldsymbol{e}_{i} g_{i}+\frac{1}{2}\left(\mathbf{F}_{S}+\mathbf{F}_{B}\right)}{\rho-d_{\phi} \rho \cdot\left(M_{\phi} \nabla \mu\right)}$, and

$\mathrm{p}=\frac{c_{S}^{2}}{1-w_{0}}\left[\sum_{i \neq 0} g_{i}+\frac{1}{2} \mathbf{u} \cdot \nabla \rho+\rho s_{0}(\mathbf{u})\right]$.

Density has a linear relationship with the order parameter, calculated as 
$\rho=\rho_{g}+\frac{\phi-\phi_{l}}{\phi_{l}-\phi_{g}}\left(\rho_{l}-\rho_{g}\right)$.

It is noted that by applying a matched density pair throughout the whole work, the density in the whole domain is set equal, i.e., $\rho=\rho_{l}=\rho_{g}$. As a result, the density gradient vanishes as zero. In addition, the buoyant force is added separately.

In addition, from the form of the equilibrium distribution and pressure, it can be noted that the velocity-based evolution as well as the calculation of macroscopic variables of the single-phase incompressible LBM model by Guo et al. [44] are inherited by this multiphase modeling work.

In order to ensure conservative stress across the binary interface and avoid the sharp-interface limit of phase-field models, we introduce a step form of dynamic viscosity, i.e.,

$\mu_{v i s}=\left\{\begin{array}{l}\mu_{l,} \phi>0.5\left(\phi_{l}+\phi_{g}\right) \\ \mu_{g}, \phi<0.5\left(\phi_{l}+\phi_{g}\right)\end{array}\right.$.

Similar to the phase-tracking equation, the diagonal matrix $\boldsymbol{\Lambda}^{g}=\left[s_{0}^{g}, s_{1}^{g}, s_{2}^{g}, \cdots, s_{14}^{g}\right]^{T}$ contains the relaxation times, where $s_{9}^{g}=s_{10}^{g}=s_{11}^{g}=s_{12}^{g}=s_{13}^{g}=1 / \tau^{g}$.

The computational domain is partitioned into a uniform three-dimensional (3D) Cartesian grid (each node is $\left.1 \times 1 \times 1 l u^{3}\right)$. The first and second order spatial derivatives are solved using an isotropic discretization scheme, involving the nearest-neighbor nodes $[6,17,20]$, i.e.,

$\nabla \chi(\mathbf{x})=\frac{1}{c_{s}^{2} \delta x} \sum w_{i} \mathbf{e}_{i} \chi\left(\mathbf{x}+\mathbf{e}_{i} \delta t\right)$,

$\nabla^{2} \chi(\mathbf{x})=\frac{2}{c_{s}^{2} \delta x^{2}} \sum w_{i}\left[\chi\left(\mathbf{x}+\mathbf{e}_{i} \delta t\right)-\chi(\mathbf{x})\right]$

where $\chi$ can be any scalar variable appearing in the model. The temporal derivative in Eq.12 is calculated using the first-order Euler scheme, i.e.,

$\partial_{t} \chi=\frac{\chi(t)-\chi(t-\delta t)}{\delta t}$.

\section{Method validation by layered Poiseuille flow}

For immiscible binary flow driven by a constant body force between two parallel plates, an analytical solution can be derived by applying the no-slip wall boundary condition, and the continuous velocity and shear stress condition in the interfacial region [6,19]. In our 3D simulation, the liquid phase flows in the region $0<z \leq a$ and the gas phase flows in the region $-\mathrm{a} \leq \mathrm{z}<0$. No-slip boundary condition is applied at $z= \pm a$. The driving force acts along the $\mathrm{x}$ direction, and periodic condition is applied along $\mathrm{x}$ and $\mathrm{y}$ directions.

The fluid flow is governed by the following equations:

$\rho_{l} v_{l} \nabla^{2} u_{l}=G, \rho_{g} v_{g} \nabla^{2} u_{g}=G$.

The boundary conditions are

$\left\{\begin{array}{c}\left.u_{l}\right|_{y=0}=\left.u_{g}\right|_{y=0} \\ \left.\rho_{l} v_{l}\left(\partial_{y} u_{l}\right)\right|_{y=0}=\left.\rho_{g} v_{g}\left(\partial_{y} u_{g}\right)\right|_{y=0} . \\ \left.u_{l}\right|_{y=a}=\left.u_{g}\right|_{y=-a}=0\end{array}\right.$

By solving Eq. 34 together with Eq. 35, the analytical velocity profiles of each region, which have parabolic forms, can be obtained. In addition, the analytical profile of the order parameter along the $\mathrm{z}$ direction follows the one-dimensional hyperbolic tangential form as given by Eq. 5 .

First, three mesh resolutions are applied for the convergence study, i.e., $128 \times 32 \times 128,192 \times 48 \times$ 192 , and $256 \times 64 \times 256$. We set the density pair $\rho_{l}=\rho_{g}=1 \mathrm{mu} / \mathrm{lu}^{3}$, the dynamic viscosity 
ratio $\mu_{l} / \mu_{g}=58$, and $\mu_{l}=0.1 \mathrm{mu} /(\mathrm{lu} \cdot \mathrm{ts})$ is also given. The constant driving force has a relationship with the central velocity [19], i.e., $u_{c}=G a^{2} /\left(\mu_{l}+\mu_{g}\right)$, and we have $u_{c}=0.001 \mathrm{lu} / \mathrm{ts}$. The mobility $M_{\phi}=1$ is applied in all cases shown in this section. Relative errors of velocity, calculated through Error_u $u=\left|1-u_{\text {simulated }} / u_{\text {analytical }}\right| \times 100 \%$, are shown in Fig. 1 for three mesh resolutions, where the maximum relative errors are $0.76 \%, 0.50 \%$, and $0.37 \%$, respectively, and even the coarsest mesh resolution is sufficient for most purposes.

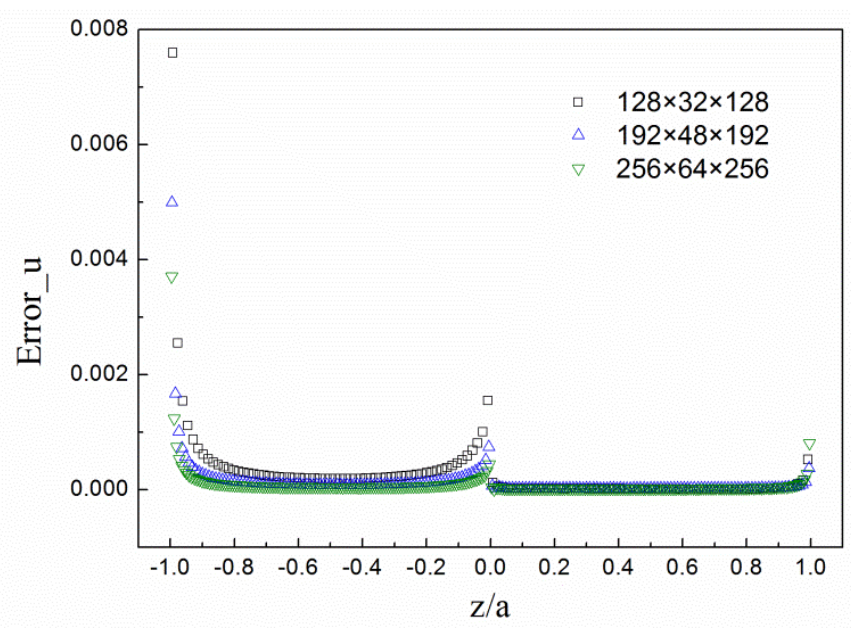

Fig. 1. Mesh convergence study for layered Poiseuille flow: relative error of velocity along the $\mathrm{z}$ direction. Three mesh resolutions along $\mathrm{x}, \mathrm{y}$, and $\mathrm{z}$ directions are $128 \times 32 \times 128,192 \times 48 \times 192$, and $256 \times 64 \times 256$, respectively.

In order to present the performance of the step-formed dynamic viscosity scheme (denoted by Scheme I), we also compare the results with the continuous scheme proposed with a linear form as $\mu=\mu_{g}+$ $\frac{\phi-\phi_{l}}{\phi_{l}-\phi_{g}}\left(\mu_{l}-\mu_{g}\right)$ (denoted as Scheme II). Results are shown in Fig. 2 and Fig. 3. It can be noted that both schemes show good results of the order parameter profile. With Scheme II, the velocity profile becomes continuous in the interfacial region, which deviates from the sharp analytical result. Meanwhile, by using Scheme I, the simulated velocity fits the analytical results well. Therefore, our step-formed dynamic viscosity shows better agreement with the analytical solution.

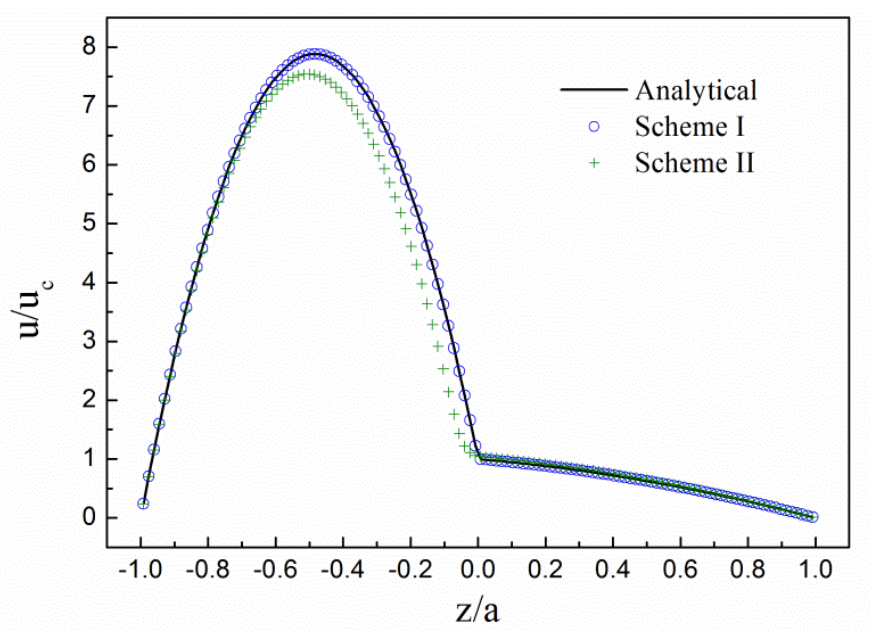

Fig. 2. Velocity profile along the $\mathrm{z}$ direction of layered Poiseuille flow, with mesh resolution of $128 \times 32 \times 128$. 


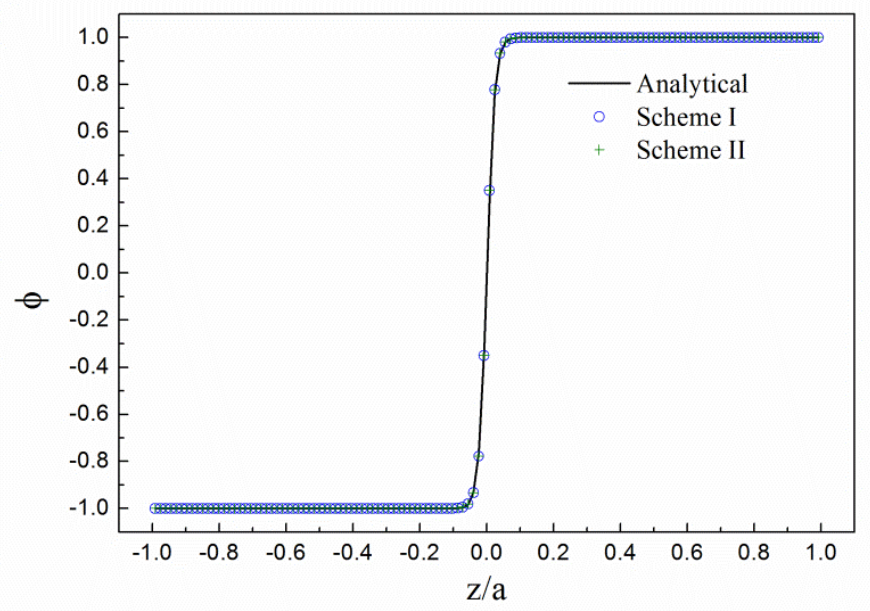

Fig. 3. Order parameter profile along the $\mathrm{z}$ direction of layered Poiseuille flow, with mesh resolution of $128 \times 32 \times 128$.

\section{Results and discussion}

\subsection{Computational setup}

For the rising process of a single bubble, the dynamic behavior is dominated by buoyancy, interfacial tension and viscous stress. In addition to the Péclet number and the Cahn number defined in Sec 2.1, we apply the following non-dimensional parameters to characterize the flow regime and hydrodynamic properties, including the Eötvös number (Eo), the Morton number (Mo), and the Reynolds number (Re), defined as

$E o=\frac{g \rho_{l} d_{0}^{2}}{\sigma}$

$M o=\frac{g \mu_{l}^{4}}{\rho_{l} \sigma^{3}}$

$R e=\frac{\rho_{l} V d_{0}}{\mu_{l}}$,

where $g$ is the gravitational acceleration, $\rho_{l}$ is the density of the liquid phase, $d_{0}$ is the initial diameter of the bubble, $\sigma$ is surface tension, and $V$ is the average velocity of the bubble.

It is noted that in some literature citations, the density difference, i.e., $\Delta \rho=\rho_{l}-\rho_{g}$, is applied in the definition of Eo and Mo. Herein the main objective is to be consistent with the experimental work of Clift et al. [1] and Bhaga et al. [2], and it is assumed that $\rho_{l} \gg \rho_{g}$ therein.

In addition, reference length $x^{*}=d_{0}$ and reference time $t^{*}=\sqrt{d_{0} / g}$ are chosen, and the definitions of the Péclet number and the Cahn number become

$P e=\frac{d_{0}^{2} \sqrt{d_{0} g}\left(\phi_{l}-\phi_{g}\right)^{2}}{M_{\phi} \sigma}$,

$C h=\frac{W}{d_{0}}$.

In the phase-field model, the density ratios of liquid/gas can be arbitrary, but numerical instability would occur if large density ratios are adopted. In our experience, the achievable density ratio is usually less than 10. In this article, we simulate using a matched density pair, i.e., $\rho_{l} / \rho_{g}=1$ and incorporate buoyant force separately. Inspired by the fact that in the layered Poiseuille flow, both the shear stress and the streamwise velocity are continuous across the interface, and the dynamic viscosity ratio plays a critical role, we also apply a high dynamic viscosity, i.e., $\mu_{l} / \mu_{g}=58$, in our simulations. Moreover, when a matched density pair is used in the simulations, the artificial interfacial force in Eq. 20 is eliminated.

In terms of boundary configuration, we apply periodic boundary conditions in the $x$ and $y$ horizontal 
directions, and no-slip wall boundary condition in the $z$ direction (direction of buoyant force). A no-flux boundary condition is also applied to the wall boundary, with $\phi$ and $\mu$ equaling the values of their neighboring fluid node, respectively.

Initially, the bubble is set to be of spherical shape, with a diffusive layer following the one-dimensional interface profile in Eq. 5. The initial diameter of the bubble is $d_{0}=50 \mathrm{lu}$.

\subsection{Terminal shape and velocity}

Among the factors that dominate the terminal shape of the bubble, interfacial tension tends to promote the smallest surface area, i.e., circle in 2D and sphere in 3D; dynamic viscosity dominates the shear stress, thus determining the rate of approach to equilibrium and influencing drag force; buoyancy acts as the driving force and equals the value of drag force in the equilibrium state.

To validate our numerical results and make comparisons with experimental measurements, we follow the experimental studies of Clift et al. [1] and Bhaga et al. [2] with equal initial values of Eo and Mo. Herein we use three groups of simulation cases: Case A $(E 0=116, M o=266)$, Case $B(E o=116, M o=41.1)$, and Case $\mathrm{C}(\mathrm{Eo}=116, \mathrm{Mo}=5.51)$. Different from Eo and Mo that measure interaction evolved in the momentum equation, the Péclet number considers the effect of mobility in the phase-tracking equation. In this section, we simulate situations with a constant mobility $M_{\phi}=22.25$, thus the Péclet number is $P e_{0}=44,194$. A constant interface, i.e., $W=3 l u$ is also used, thus the Cahn number is $C h=0.06$. Table 1 lists the terminal Reynolds number simulated using different mesh resolutions in both the streamwise and spanwise directions, previous experimental results by Clift et al. [1], Bhaga et al. [2], and numerical results by Hua et al. [27], and Cheng et al. [29]. It is clear that the present results fit the experimental results quite well. Snapshots of terminal bubble shape in the 3D domain and comparisons with experimental results [2] are shown in Fig. 4.

Table 1 Comparisons of terminal Reynolds number and mesh convergence study

\begin{tabular}{|c|c|c|c|c|c|c|c|c|c|c|}
\hline \multirow[b]{2}{*}{ Case } & \multicolumn{2}{|c|}{ Experimental } & \multicolumn{2}{|c|}{ Numerical } & \multicolumn{6}{|c|}{ Present study with different mesh resolutions } \\
\hline & $\begin{array}{l}\text { Clift } \\
\text { et al. }\end{array}$ & $\begin{array}{l}\text { Bhaga } \\
\text { et al. }\end{array}$ & $\begin{array}{l}\text { Hua } \\
\text { et al. }\end{array}$ & $\begin{array}{l}\text { Cheng } \\
\text { et al. }\end{array}$ & $160 \times 160 \times 352$ & $192 \times 192 \times 320$ & $192 \times 192 \times 352$ & $192 \times 192 \times 384$ & $224 \times 224 \times 352$ & $256 \times 256 \times 352$ \\
\hline A & 3.48 & 3.57 & 3.62 & 3.62 & 3.404 & 3.464 & 3.465 & 3.465 & 3.479 & 3.482 \\
\hline $\mathrm{B}$ & 6.81 & 7.16 & 7.00 & 7.20 & 6.702 & 6.823 & 6.825 & 6.825 & 6.857 & 6.861 \\
\hline $\mathrm{C}$ & 13.0 & 13.3 & 13.2 & 13.7 & 12.71 & 12.95 & 12.96 & 12.96 & 13.03 & 13.04 \\
\hline
\end{tabular}

From Table 1, firstly, in the flow direction, the mesh with NZ $=352$ is sufficient for all three cases. In the horizontal direction, we note that the terminal Reynolds number is a bit influenced by the mesh resolution, however, good convergence is reached when the mesh resolution is NX $\times \mathrm{NY} \times \mathrm{NZ}=192 \times$ $192 \times 352$, and compared to the results with finest mesh resolution, i.e., $\mathrm{NX} \times \mathrm{NY} \times \mathrm{NZ}=256 \times 256 \times$ 352 , the relative error is close to $0.5 \%$. Through the convergence study, it is shown that the mesh resolution with $\mathrm{NX} \times \mathrm{NY} \times \mathrm{NZ}=192 \times 192 \times 352$ is sufficient and appropriate for simulating the cases. Moreover, the terminal velocities of Case A, Case B, and Case C are $0.00792 \mathrm{lu} / \mathrm{ts}, 0.00978 \mathrm{lu} / \mathrm{ts}$, and $0.0112 \mathrm{lu} / \mathrm{ts}$, respectively, indicating that the incompressible limit $u_{t} \ll c_{s}$ is satisfied well. 


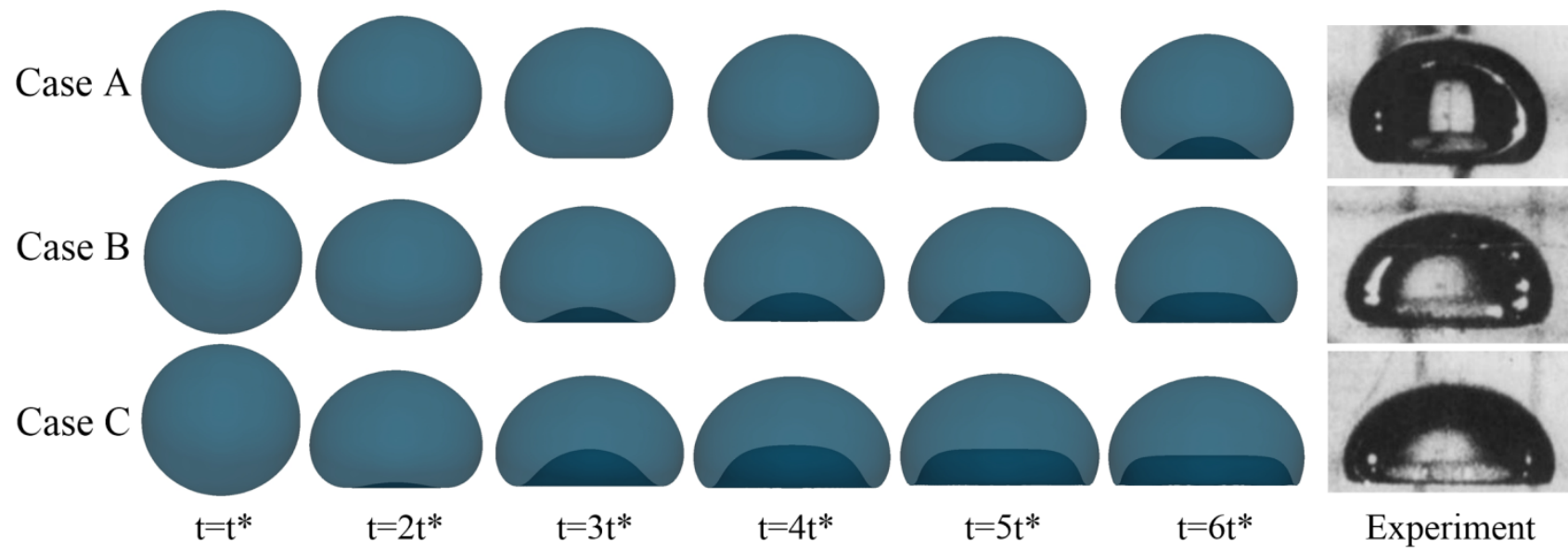

Fig. 4. Time evolution of bubble shape and comparisons with experimental results (Case A: Eo=116, Mo=266; Case B: $\mathrm{Eo}=116, \mathrm{Mo}=41.1$; Case $\mathrm{C}: \mathrm{Eo}=116, \mathrm{Mo}=5.51)$. Experimental results are from Bhaga et al.

In each case, average velocity and centroid position are monitored every 100 lattice time steps $\left(0.04 t^{*}\right)$, and are calculated as

$u_{\text {average }, z}=\frac{\sum \rho_{i} u_{i, z} s\left(\phi_{i}\right)}{\sum \rho_{i} s\left(\phi_{i}\right)}$

$Z c_{\text {average }, z}=\frac{\sum \rho_{i} Z_{i} s\left(\phi_{i}\right)}{\sum \rho_{i} s\left(\phi_{i}\right)}$

where $s\left(\phi_{i}\right)$ is a switch function, i.e., $s\left(\phi_{i}\right)=1$ when $\phi_{i}<\left(\phi_{l}+\phi_{g}\right) / 2$, and indicates that the node is inside the bubble; or $s\left(\phi_{i}\right)=0$ when $\phi_{i}>\left(\phi_{l}+\phi_{g}\right) / 2$. The time evolution of the Reynolds number according to average bubble velocity, and the centroid position are shown in Fig. 5 and Fig. 6, respectively.

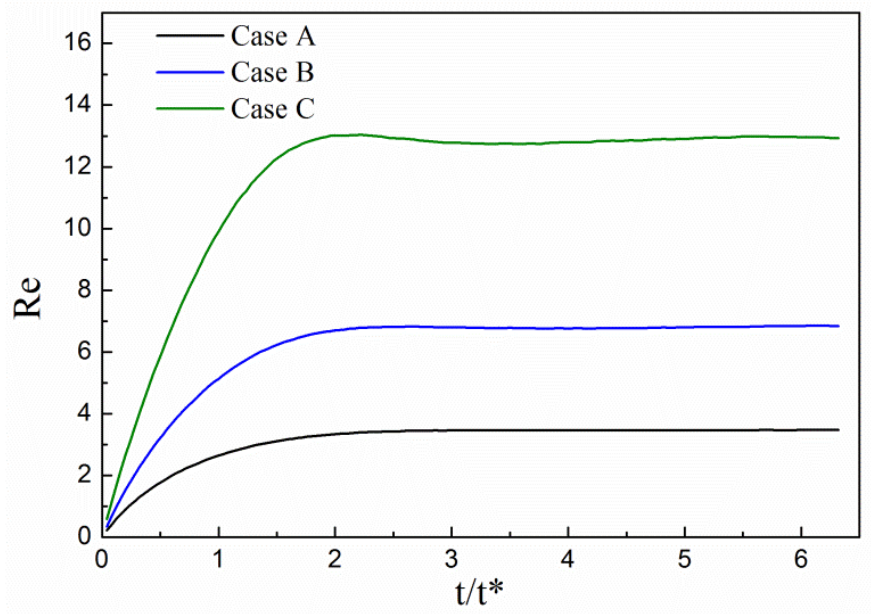

Fig. 5. Time evolution of Reynolds number at $\mathrm{Pe}=44,194$ and $\mathrm{Ch}=0.06$. Case $\mathrm{A}$ : Eo=116, Mo=266; Case $\mathrm{B}$ : Eo=116, $\mathrm{Mo}=41.1$; Case $\mathrm{C}: \mathrm{Eo}=116, \mathrm{Mo}=5.51$. 


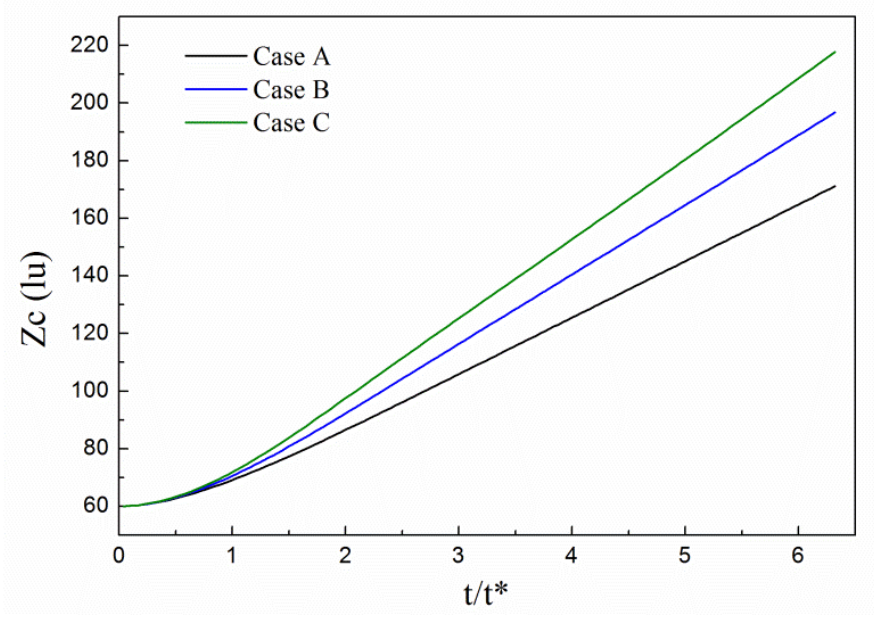

Fig. 6. Time evolution of bubble centroid position at $\mathrm{Pe}=44,194$ and $\mathrm{Ch}=0.06$. Case $\mathrm{A}$ : $\mathrm{E} o=116, \mathrm{Mo}=266$; Case $\mathrm{B}$ : Eo=116, $\mathrm{Mo}=41.1$; Case $\mathrm{C}: \mathrm{Eo}=116, \mathrm{Mo}=5.51$.

In addition, drag force acting on the bubble is evaluated indirectly by measuring the momentum change during fixed intervals, i.e.

$F_{\text {drag }, t}=F_{B}-\frac{1}{n \delta t}\left[\sum_{t+n \delta t / 2} \rho_{i} u_{i, z} s\left(\phi_{i}\right)-\sum_{t-n \delta t / 2} \rho_{i} u_{i, z} s\left(\phi_{i}\right)\right]$,

where $n=100$ is the statistical interval. Time evolution of calculated drag force is shown in Fig. 7. The final drag force of the bubble reaches its equilibrium value after approximately $3 t^{*}$ time steps. Before that, it keeps changing as it rises and deforms towards the terminal shape. During that period of time, on one hand, smaller drag force will be acting on the bubble in cases where smaller viscosity is applied; on the other hand, with smaller drag force, the net force acting on the bubble will be larger, bringing a larger rate of deformation.

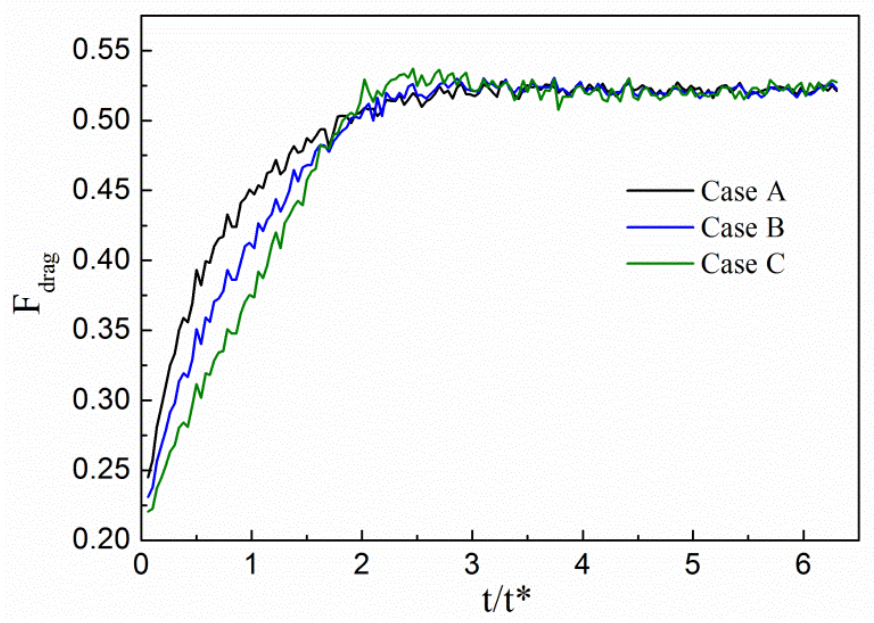

Fig. 7. Time evolution of drag force acting on the bubble at $\mathrm{Pe}=44,194$. Case A: Eo=116, Mo=266; Case B: Eo=116, $\mathrm{Mo}=41.1$; Case $\mathrm{C}: \mathrm{Eo}=116, \mathrm{Mo}=5.51$.

\subsection{Effect of mobility and interface width}

Since it is still an open problem how to choose the value of mobility based on physical principles, it is important to explore the effect of mobility numerically before performing simulations. According to the $\mathrm{CH}$ equation and the definition of chemical potential, for a given flow regime, a relatively small value of mobility would result in a small rate of diffusion. In addition, based on the LB approach, the achievable value of mobility is often limited to a certain range, and numerical instability would occur beyond this range. 
It has been reported recently that mobility has a close relationship with the interface width [14,45]. It is suggested that a value of mobility that is proportional to the square of the interface width is optimal for achieving thin interfaces. Since this conclusion is a general one, in specific problems, it is essential to identify an appropriate range of the mobility parameter.

Firstly, we conduct a rising bubble study to test the effect of interface width with a fixed value of mobility, and the results are shown in Fig. 8. The relative error in terminal Reynolds number compared with the case at $\mathrm{W}=3 \mathrm{lu}$ is less than $1.01 \%$. Hence only minor influence of interface width on convergence to a constant Reynolds number is observed. Based on this study, we can conclude that interface width is not a major factor affecting the hydrodynamics of the rising bubble. At least at the interface width range of $\mathrm{W}=2-4 \mathrm{lu}$, this justifies further consideration of the effect of mobility independent of the interface width.

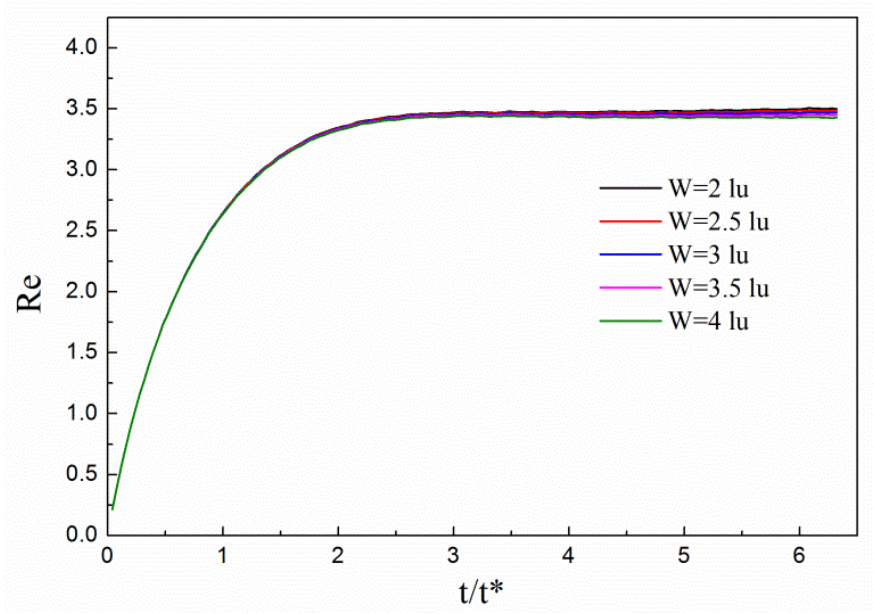

Fig. 8. Terminal Reynolds number with different interface width. (Case A: Eo=116, Mo=266)

For the cases investigated, where the value of Reynolds number indicates a moderate flow regime, the effect of mobility on the bubble shape is minor when the shape is drawn simply with the surface where the order parameter equals to $\left(\phi_{l}+\phi_{g}\right) / 2$. However, previous work has seldom presented the spatial distribution of the density or order parameter. To make comparisons, Fig. 9 11 show terminal bubble shapes (center cross section along $y$ direction) with different values of Pe in Cases A, B, and C, each drawn by 11 equal-interval contours of order parameter ranging from -0.5 to 0.5 .
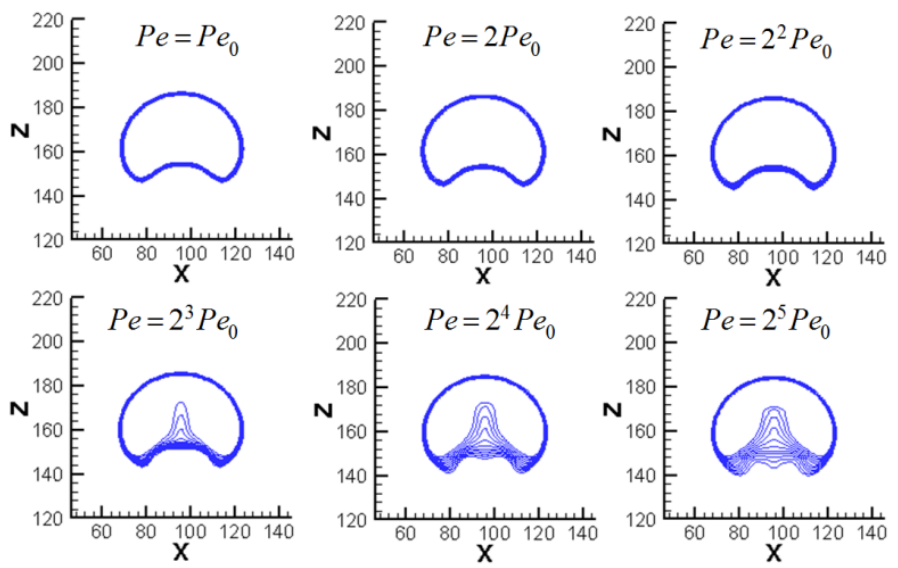

Fig. 9. Terminal shape with different mobility. (Case A: Eo=116, Mo=266) 

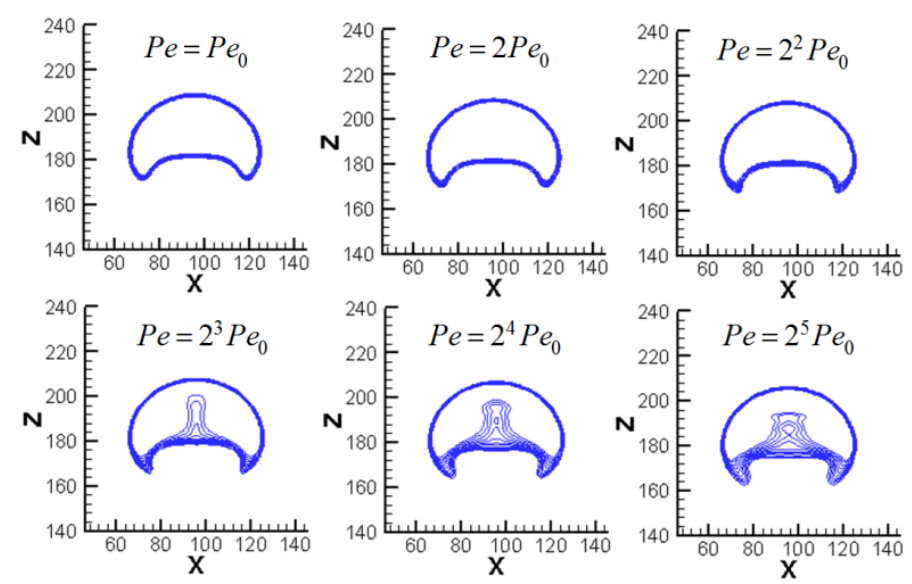

Fig. 10. Terminal shape with different mobility. (Case B: Eo=116, Mo=41.1)
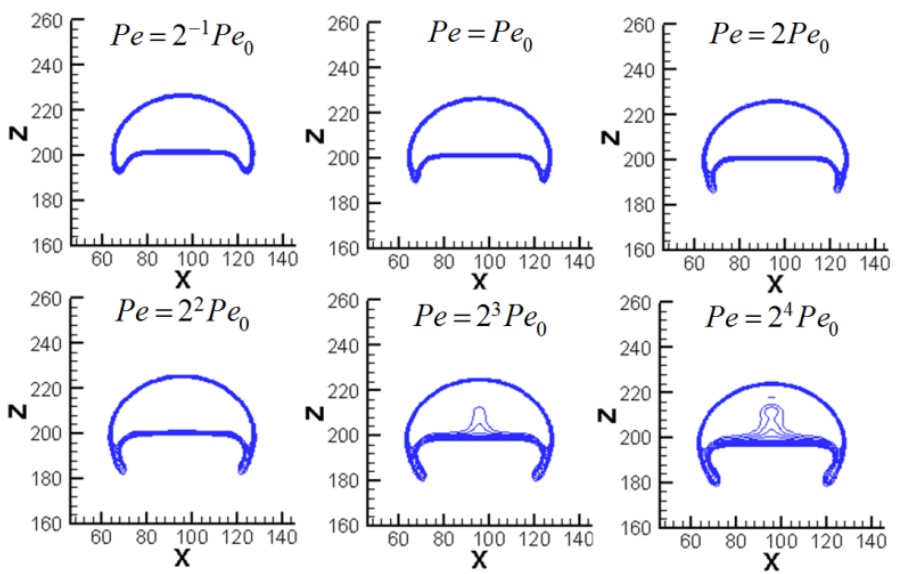

Fig. 11. Terminal shape with different mobility. (Case C: $E 0=116, M o=5.51)$

As the bubble rises, the shape deforms from its initial spherical shape and the lower part tends to shrink due to the wake, where the effect of mobility is significant. From Fig. 9 11, it can be noted that for relatively small values of mobility, the spatial distribution of the order parameter deviates from real situations where sharp interfaces exist. In order to obtain more detailed information on the influence of mobility on the interfaces, the minimum and maximum values of the order parameter in the whole domain are measured and are shown in Fig. 12 and Fig. 13, respectively.

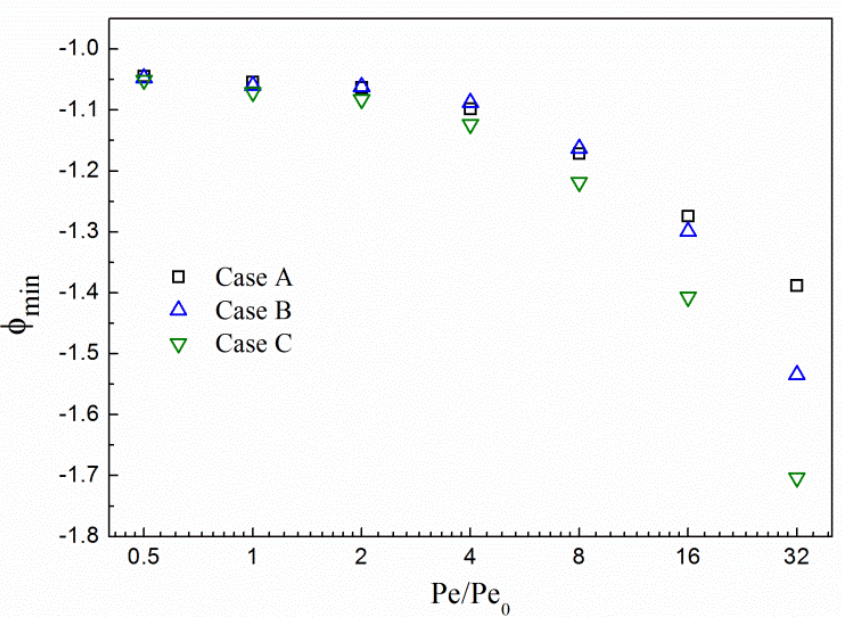

Fig. 12. Minimum value of order parameter. Case $A$ : $E o=116, M o=266$; Case $B: E o=116, M o=41.1$; Case $C$ : $E o=116$, $\mathrm{Mo}=5.51$. 


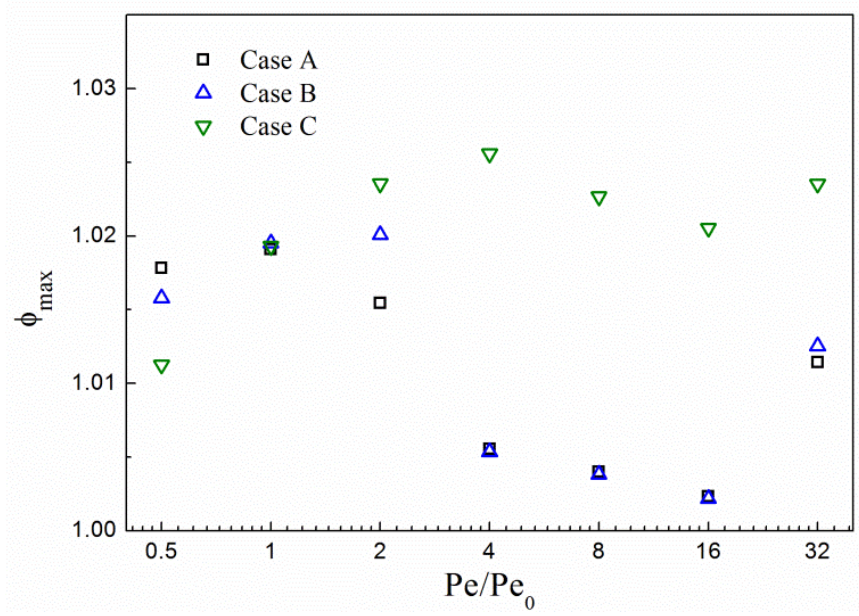

Fig. 13. Maximum value of order parameter. Case A: Eo=116, Mo=266; Case B: Eo=116, Mo=41.1; Case C: Eo=116, $\mathrm{Mo}=5.51$.

Peak values of the order parameter exist near the interface. As the value of mobility decreases (higher $\mathrm{Pe}$ ), the minimum value of order parameter decreases monotonically. And in cases where terminal velocity is larger, the minimum value of the order parameter will be decreased more. From the $\mathrm{CH}$ equation, it can be concluded that the above effect is caused by improper diffusion. In addition, the maximum value of the order parameter in the whole domain is only slightly influenced, and is close to the expected value in all cases. The above results also suggest that when conducting phase-field-based simulations, whether the value of mobility is appropriate can be checked through monitoring the maximum and minimum values of the order parameter.

Moreover, for cases with larger values of Mo, or small values of dynamic viscosity, when skirted tails or break-up appears, bifurcation induced by mobility is observed. For cases with large values of mobility, or small values of Péclet number, small bubbles appear to separate more easily in the simulations than in the experiments (see Fig. 14(b)). Meanwhile, it can be noted that the shape of the main part of the bubble is almost the same as the experimental results, and the position of the bubble is unaffected by the Péclet number, after $6 t^{*}$ time steps. Although terminal shape can be captured after sufficient time steps, the effect of an improper value of mobility on the dynamic behavior is more complex.
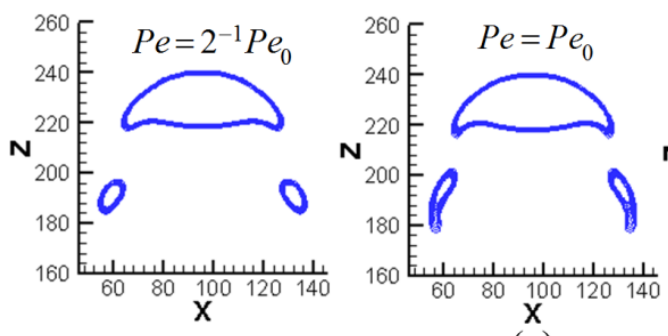

(a)

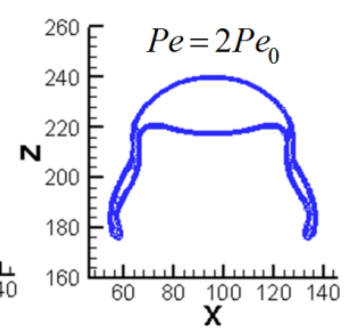

Fig. 14. Terminal shape (a) with different mobility and (b) experimental results by Bhaga et al. at Eo=116 and Mo=0.103.

\subsection{Effect of dynamic viscosity ratio}

In order to assess the importance of the dynamic viscosity ratio, in this section we perform bubble rise simulations and apply a group of dynamic viscosity ratios at Eo=116 and Mo=266. Herein we keep all parameters fixed to the same values as those of Case A in Sec. 4.2, except for the dynamic viscosity of the gas phase and thereby the dynamic viscosity ratio. The time evolution of the Reynolds number is calculated to make comparisons, and the results are shown in Fig.15, where $\mu_{r}$ denotes the dynamic viscosity ratio of liquid to gas phases. 


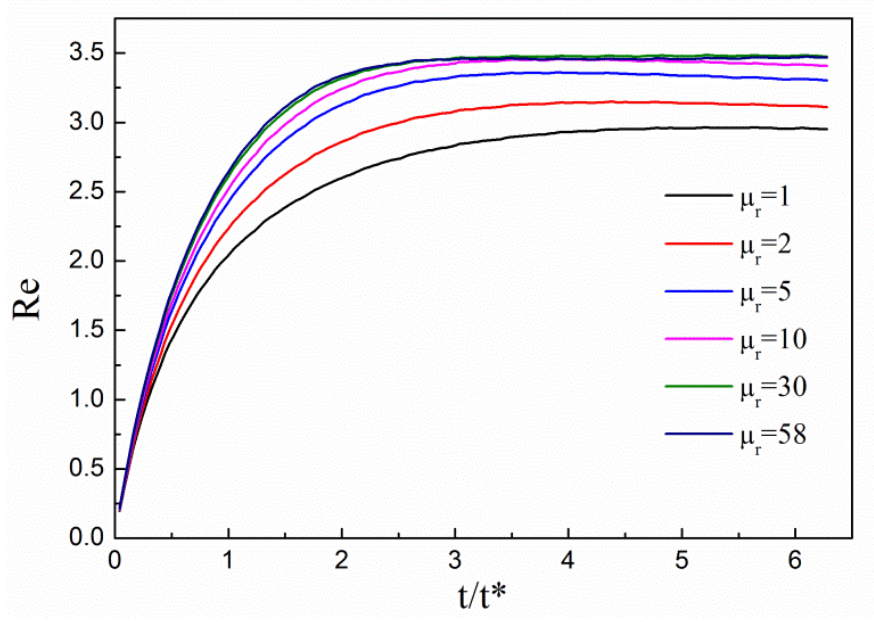

Fig. 15. Terminal Reynolds number with different dynamic viscosity ratio. (Case A: Eo=116, Mo=266, Pe=44194)

The results in Fig. 15 indicate that, for the modeled conditions (Case A), there is convergence of the Reynolds number to the experimental result $(\mathrm{Re}=3.48$ listed in Table 1$)$ for dynamic viscosity ratio greater than about 30. It is shown from Fig. 15 that at low dynamic viscosity ratios, more time steps are needed to reach the equilibrium state and the terminal velocity is smaller than experimental data. As a bubble rises at a moderate Reynolds number, the movement of the surrounding liquid phase owes much to the shear stress induced by the bubble, where the dynamic viscosity plays a significant role. In cases where large viscosity ratios are applied, the surrounding liquid phase is weakly influenced by the movement of the bubble.

Different from the intensively studied topic $[6,20,29]$ to pursue a large density ratio in multiphase modeling, our simulations show that a correct treatment of dynamic viscosity across the interfacial region is also an important issue. A benefit of the current model is that it can achieve a very low viscosity in practical problems, both a high viscosity ratio and a relatively high Reynolds number can be achieved concurrently.

\section{Conclusions}

In this article, we present a numerical study of terminal shape and velocity of a rising bubble in various flow regimes, with an emphasis on the effect of the Cahn-Hilliard mobility. Based on the latest phase field model developed by Liang et al. [17], the incompressible multiphase flow of a rising bubble, including the phase-tracking and hydrodynamic equations, is fully evolved in a three-dimensional domain. Unlike previous numerical simulations, we apply a matched density pair, a high dynamic viscosity ratio, effective buoyancy, and a modified interfacial tension calculation scheme.

Using the LB model, the terminal shape and velocity of the rising bubble are in good agreement with previous experimental and numerical results. With constant Eo and Mo that dominate the dynamic process and various values of mobility, it is noted that large values of mobility maintain relatively sharp interfaces; while smaller values of mobility would result in non-physical diffusive interfacial regions. Moreover, for cases with large Reynolds number, small bubbles at the tail tend to separate more easily when the value of mobility is larger. Since the physical meaning of mobility is not yet fully understood, bifurcation induced by mobility should be noted and explored carefully.

In addition, simulations concerning the effect of dynamic viscosity show that a low dynamic viscosity would result in a considerably slower rate of evolution towards the equilibrium state, as well as deviated terminal velocity. A dynamic viscosity ratio that approaches the real system is recommended.

Overall, our work reveals good capability of the incompressible phase-field-based model, and offers useful tests concerning the effect of mobility.

\section{Acknowledgements}

This article is supported by the Young Scientists Fund of the National Science Foundation of China 
(Grant No. 11502210), Natural Science Basic Research Plan in Shaanxi Province of China (Grant No. 2016JM1002), Natural Science Basic Research Plan in Shenzhen City of China (Grant No. JCYJ20160510140747996), and the Innovation Foundation for Doctoral Dissertation of Northwestern Polytechnical University (Grant No. CX201501). The first author receives financial funding from China Scholarship Council. This material is also based upon work supported by the National Science Foundation under Grant No. EAR-1204762. The authors acknowledge the Instructional \& Research Computing Center at Florida International University for providing the High Performance Computing (HPC) resource, and we are grateful for assistance from Dr. Mengxing Cheng, the HPC system administrator. The authors also appreciate the comments and suggestions of the editor and the referees that substantially improved this article.

\section{References}

[1] Clift R, Grace JR, Weber ME. Bubbles, Drops and Particles. New York: Academic Press; 1978.

[2] Bhaga D, Weber ME. Bubbles in viscous liquids: shapes, wakes and velocities. J Fluid Mech 1981;105:61-85. doi:10.1017/S002211208100311X.

[3] Corapcioglu MY. Rise velocity of an air bubble in porous media: Theoretical studies. Water Resour Res 2004;40:1-9. doi:10.1029/2003WR002618.

[4] Maxworthy T, Gnann C, Kürten M, Durst F. Experiments on the rise of air bubbles in clean viscous liquids. J Fluid Mech 1996;321:421-41. doi:10.1017/S0022112096007781.

[5] Sukop MC, Thorne DT. Lattice boltzmann modeling: An introduction for geoscientists and engineers. Springer Berlin Heidelberg; 2006.

[6] Haibo H, Sukop MC, Lu X-Y. Multiphase Lattice Boltzmann Methods. Wiley; 2015.

[7] Scardovelli R, Zaleski S. Direct Numerical Simulation of Free-Surface and Interfacial Flow. Annu Rev Fluid Mech 1999;31:567-603. doi:10.1146/annurev.fluid.31.1.567.

[8] Hirt CW, Nichols BD. Volume of fluid (VOF) method for the dynamics of free boundaries. J Comput Phys 1981;39:201-25. doi:10.1016/0021-9991(81)90145-5.

[9] Adalsteinsson D, Sethian J a. A fast level set method for propagating interfaces. J Comput Phys 1995;118:269-77.

[10] Caginalp G. An analysis of a phase field model of a free boundary. Arch Ration Mech Anal 1986;92:205-45. doi:10.1007/BF00254827.

[11] Kim J. Phase-Field Models for Multi-Component Fluid Flows. Commun Comput Phys 2012;12:613-61. doi:10.4208/cicp.301110.040811a.

[12] Cahn JW, Elliott CM, Novick-Cohen A. The Cahn-Hilliard equation with a concentration dependent mobility: motion by minus the Laplacian of the mean curvature. Eur J Appl Math 1996;7:1-16. doi:10.1017/S0956792500002369.

[13] Huang JJ, Shu C, Chew YT. Mobility-dependent bifurcations in capillarity-driven two-phase fluid systems by using a lattice Boltzmann phase-field model. Int J Numer Methods Fluids 2009;60:203-25.

[14] Magaletti F, Picano F, Chinappi M, Marino L, Casciola CM. The sharp-interface limit of the Cahn-Hilliard/NavierStokes model for binary fluids. J Fluid Mech 2013;714:95-126. doi:10.1017/jfm.2012.461.

[15] Mattiussi C. An Analysis of Finite Volume , Finite Element , and Finite Difference Methods Using Some Concepts from Algebraic Topology. J Comput Phys 1997;133:289-309.

[16] Zheng HW, Shu C, Chew YT. A lattice Boltzmann model for multiphase flows with large density ratio. J Comput Phys 2006;218:353-71. doi:10.1016/j.jcp.2006.02.015.

[17] Liang H, Shi BC, Guo ZL, Chai ZH. Phase-field-based multiple-relaxation-time lattice Boltzmann model for incompressible multiphase flows. Phys Rev E 2014;89:055320. doi:10.1103/PhysRevE.89.053320.

[18] Liang H, Chai ZH, Shi BC, Guo ZL, Zhang T. Phase-field-based lattice Boltzmann model for axisymmetric multiphase flows. Phys Rev E 2014;90:063311. doi:10.1103/PhysRevE.90.063311.

[19] Zu YQ, He S. Phase-field-based lattice Boltzmann model for incompressible binary fluid systems with density and viscosity contrasts. Phys Rev E 2013;87:043301. doi:10.1103/PhysRevE.87.043301. 
[20] Shao JY, Shu C. A hybrid phase field multiple relaxation time lattice Boltzmann method for the incompressible multiphase flow with large density contrast. Int J Numer Methods Fluids 2015;77:526-43. doi:10.1002/fld.3995.

[21] Rothman DH, Keller JM. Immiscible cellular-automaton fluids. J Stat Phys 1988;52:1119-27. doi:10.1007/BF01019743.

[22] Shan X, Chen H. Lattice Boltzmann model for simulating flows with multi phases and components. Phys Rev E 1993;47:1815-9. doi:10.1103/PhysRevE.47.1815.

[23]Chen L, Kang Q, Mu Y, He YL, Tao WQ. A critical review of the pseudopotential multiphase lattice Boltzmann model: Methods and applications. Int J Heat Mass Transf 2014;76:210-36. doi:10.1016/j.ijheatmasstransfer.2014.04.032.

[24] Michael Swift, W osborn, Yeomans JM. Lattice Boltzmann Simulation of Nonideal Fluids. Phys Rev Lett 1995;75:830-3.

[25] He X, Chen S, Zhang R. A Lattice Boltzmann Scheme for Incompressible Multiphase Flow and Its Application in Simulation of Rayleigh-Taylor Instability. J Comput Phys 1999;152:642-63. doi:10.1006/jcph.1999.6257.

[26] Zhang R, He X, Doolen G, Chen S. Surface tension on two-dimensional two-phase Kelvin-Helmholtz instabilities. Adv Water Resour 2001;24:461-78.

[27] Hua J, Stene JF, Lin P. Numerical simulation of 3D bubbles rising in viscous liquids using a front tracking method. J Comput Phys 2008;227:3358-82. doi:10.1016/j.jcp.2007.12.002.

[28] Hua J, Lou J. Numerical simulation of bubble rising in viscous liquid. J Comput Phys 2007;222:769-95. doi:10.1016/j.jcp.2006.08.008.

[29] Cheng M, Hua J, Lou J. Simulation of bubble-bubble interaction using a lattice Boltzmann method. Comput Fluids 2010;39:260-70. doi:10.1016/j.compfluid.2009.09.003.

[30] Huang H, Huang J-J, Lu X-Y. A mass-conserving axisymmetric multiphase lattice Boltzmann method and its application in simulation of bubble rising. J Comput Phys 2014;269:386-402. doi:10.1016/j.jcp.2014.03.028.

[31] Ngachin M, Galdamez RG, Gokaltun S, Sukop MC. Lattice Boltzmann simulation of rising bubble dynamics using an effective buoyancy method. Int J Mod Phys C 2014;26:1550031. doi:10.1142/S012918311550031X.

[32] Amaya-Bower L, Lee T. Single bubble rising dynamics for moderate Reynolds number using Lattice Boltzmann Method. Comput Fluids 2010;39:1191-207. doi:10.1016/j.compfluid.2010.03.003.

[33] Fakhari A, Rahimian MH. Simulation of falling droplet by the lattice Boltzmann method. Commun Nonlinear Sci Numer Simul 2009;14:3046-55. doi:10.1016/j.cnsns.2008.10.017.

[34] Fakhari A, Rahimian MH. Investigation of deformation and breakup of a moving droplet by the method of lattice Boltzmann equations. Int J Numer Methods Fluids 2010;64:827-49. doi:10.1002/fld.2172.

[35] Chao J, Mei R, Singh R, Shyy W. A filter-based, mass-conserving lattice Boltzmann method for immiscible multiphase flows. Int J Numer Methods Fluids 2011;66:622-47.

[36] Wang C, Khoo BC. An indirect boundary element method for three-dimensional explosion bubbles. J Comput Phys 2004;194:451-80. doi:10.1016/j.jcp.2003.09.011.

[37] Klaseboer E, Turangan C, Fong SW, Liu TG, Hung KC, Khoo BC. Simulations of pressure pulse-bubble interaction using boundary element method. Comput Methods Appl Mech Eng 2006;195:4287-302. doi:10.1016/j.cma.2005.08.014.

[38] Zhang AM, Liu YL. Improved three-dimensional bubble dynamics model based on boundary element method. J Comput Phys 2015;294:208-23. doi:http://dx.doi.org/10.1016/j.jcp.2015.03.049.

[39] Premnath KN, Abraham J. Three-dimensional multi-relaxation time (MRT) lattice-Boltzmann models for multiphase flow. J Comput Phys 2007;224:539-59. doi:10.1016/j.jcp.2006.10.023.

[40]Lallemand P, Luo L-S. Theory of the lattice Boltzmann Method: Dispersion, Dissipation, Isotropy, Galilean Invariance, and Stability. Phys Rev E 2000;61:6546-62. doi:10.1103/PhysRevE.61.6546.

[41] Premnath KN, Pattison MJ, Banerjee S. Generalized lattice Boltzmann equation with forcing term for computation of wall-bounded turbulent flows. Phys Rev E 2009;79:026703. doi:10.1103/PhysRevE.79.026703.

[42] Li Q, Luo KH, Gao YJ, He YL. Additional interfacial force in lattice Boltzmann models for incompressible multiphase flows. Phys Rev E 2012;85:026704. doi:10.1103/PhysRevE.85.026704. 
[43]Kim J. A continuous surface tension force formulation for diffuse-interface models. J Comput Phys 2005;204:784-804. doi:10.1016/j.jcp.2004.10.032.

[44] Guo Z, Shi B, Wang N. Lattice BGK Model for Incompressible Navier-Stokes Equation. J Comput Phys 2000;165:288-306. doi:10.1006/jcph.2000.6616.

[45] Yue P, Zhou C, Feng JJ. Sharp-interface limit of the Cahn-Hilliard model for moving contact lines. J Fluid Mech 2010;645:279. doi:10.1017/S0022112009992679. 